\title{
Electroosmotic flow and dispersion in open and closed porous media
}

\section{Authors: Robert S. Maier, Elmira Nybo, Joseph D. Seymour, \& Sarah L. Codd}

NOTICE: The final publication is available at Springer via http://dx.doi.org/10.1007/s11242-016-0680-4.

Maier RS, Nybo E, Seymour JD, Codd SL, "Electroosmotic flow and dispersion in open and closed porous media," Transport in Porous Media, 2016 May 113(1): 67-89. 


\title{
Electroosmotic Flow and Dispersion in Open and Closed Porous Media
}

\author{
Robert S. Maier ${ }^{1}$. Elmira Nybo 1 . \\ Joseph D. Seymour ${ }^{1} \cdot$ Sarah L. Codd $^{2}$
}

1 Department of Chemical and Biological Engineering, Montana State University, Bozeman, MT, USA

2 Department of Mechanical and Industrial Engineering, Montana State University, Bozeman, MT, USA

Abstract Electroosmotic flow and dispersion in open and closed packed beds were inves-tigated using Nuclear Magnetic Resonance (NMR) spectroscopy and pore-scale simulation. A series of NMR spectroscopy experiments were conducted to measure the effect of elec-troosmotic pressure on dispersion in packed spheres as a function of diameter and electric field strength. The experiments confirm earlier observations by others of superdiffusive trans-port in closed media. However, superdiffusive behavior is observed even at small pore sizes, contrary to earlier results and simulations in fixed sphere packs, and is conjectured to result from pressure-induced rearrangement of the particles. Simulations also support the existence of pore size-independent velocity distributions in closed media. The distribution of reverse velocities is also similar, apart from a difference in sign, to pressure-driven flow in open porous media.

Keywords Electroosmosis $\cdot$ Dispersion $\cdot \mathrm{NMR} \cdot$ Pore-scale $\cdot$ Lattice-Boltzmann

\section{List of Symbols}

$\begin{array}{ll}\boldsymbol{v}_{\boldsymbol{S}} & \text { Slip velocity }(\mathrm{m} / \mathrm{s}) \\ \overline{\boldsymbol{v}} & \text { Average pore velocity }(\mathrm{m} / \mathrm{s}) \\ \boldsymbol{D}, \boldsymbol{D}(\boldsymbol{t}) & \text { Dispersion tensor }\left(\mathrm{m}^{2} / \mathrm{s}\right), \text { also restricted diffusion } \\ \boldsymbol{D}_{\boldsymbol{m}}, \boldsymbol{D}_{\mathbf{0}} & \text { coefficient Free diffusion coefficient }\left(\mathrm{m}^{2} / \mathrm{s}\right) \\ \boldsymbol{D}_{\boldsymbol{L}} & \text { Longitudinal dispersion coefficient }\left(\mathrm{m}^{2} / \mathrm{s}\right) \\ \boldsymbol{D}_{\boldsymbol{T}} & \text { Transverse dispersion coefficient }\left(\mathrm{m}^{2} / \mathrm{s}\right) \\ \boldsymbol{\tau} & \text { Tortuosity (dimensionless) }\end{array}$




\begin{tabular}{|c|c|}
\hline$L$ & Length of the domain (m) \\
\hline$d$ & Particle diameter $(\mathrm{m})$ \\
\hline$\varepsilon$ & Porosity (dimensionless) \\
\hline$\sigma_{\text {eff }}$ & Effective conductivity (S/m) \\
\hline$E$ & Electric field (V/m), applied or local \\
\hline$A$ & Cross-sectional area of the porous medium $\left(\mathrm{m}^{2}\right)$ \\
\hline$\sigma_{f}$ & Fluid conductivity $(\mathrm{S} / \mathrm{m})$ \\
\hline$\mu_{0}$ & Electrophoretic mobility (Pas) \\
\hline$\epsilon_{0}$ & Absolute permittivity $(\mathrm{F} / \mathrm{m})$ \\
\hline$\epsilon_{r}$ & Relative permittivity (dimensionless) \\
\hline$Q$ & Volumetric flow rate $\left(\mathrm{m}^{3} / \mathrm{s}\right)$ \\
\hline$K_{e}$ & Electroosmotic permeability $\left(\mathrm{m}^{2} /(\mathrm{V} \mathrm{s})\right)$ \\
\hline$K_{h}$ & Hydraulic permeability $\left(\mathrm{m}^{2}\right)$ \\
\hline$\nabla p$ & Pressure gradient $(\mathrm{Pa} / \mathrm{m})$ \\
\hline$g$ & NMR gradient pulse amplitude (T/m) \\
\hline$\delta$ & Duration of the gradient pulse (ms) \\
\hline$\Delta$ & Separation time between applied gradient pulses (ms) \\
\hline$\phi$ & Electrical potential $(\mathrm{V})$ \\
\hline$\nabla \phi$ & Potential gradient $(\mathrm{V} / \mathrm{m})$ \\
\hline$F$ & Body force $\left(\mathrm{kg} \mathrm{m} / \mathrm{s}^{2}\right)$ \\
\hline$V_{f}$ & Total electrical flux (V m) \\
\hline$\mu$ & Dynamic viscosity (Pa s) \\
\hline$p$ & Pressure $(\mathrm{Pa})$ \\
\hline$\Delta x$ & Numerical grid resolution or lattice cell size (m) \\
\hline$P e$ & Peclet number (dimensionless), $P e \equiv \bar{v} d / D_{m}$ \\
\hline$f(v)$ & Probability density function for velocity (dimensionless) \\
\hline
\end{tabular}

\section{Introduction}

Electroosmotic flow (EOF) in porous media has a variety of applications, including soil consolidation (Adamson 1966; Casagrande 1952), dewatering (Mahmoud et al. 2010), electromigration of solutes (Acar et al. 1993; Ottosen et al. 2008), particulates (Cardenas and Struble 2006; Lagerblad and Vogt 2004), and separations (Ghosal 2006). The latter applications emphasize the transport properties of EOF and depend on controlling dispersion within restricted pore spaces. EOF transport properties in porous media are also of interest in certain microfluidic applications (Ghosal 2006; Xuan et al. 2004).

An important property is that solute dispersion tends to be lower in EOF than in a pressuredriven flow (PDF) (Tallarek et al. 2000). The electroosmotic driving force occurs at the pore walls. In an open channel, the apparent slip velocity, $v_{s}$, propagates outward by viscous shear yielding a uniform(plug) flow profile, in contrast to the parabolic PDF profile. The variance of a uniform velocity distribution is minimal and because dispersion, $D(t)$, is proportional to the velocity variance (Bear 2013), dispersion tends to be lower under EOF. This behavior is exploited in electrochromatography to obtain higher separation efficiency (Ghosal 2006; Tallarek et al. 2000). Dispersion behavior is perhaps less well understood in other applications, such as electromigration, in part because media such as soils and concretes are not as well characterized as manufactured separations media. The dispersion behavior is less clear, for example, in a porous media with restrictions on flow, such as a significant 
number of blocked pores. The flow behavior is well understood in a simple closed geometry. It is well known that electroosmotic flow in a closed tube induces a reverse PDF (Marcos et al. 2005; Rice and Whitehead 1965), indicating that induced pressure gradients and reverse flows are characteristic of natural or manufactured porous media with restricted or blocked pores. There are fewer results on flow and dispersion for closed porous media. Locke et al. (2001) used Nuclear Magnetic Resonance (NMR) spectroscopy to recover the velocity distributions in open and closed cylinders packed with glass spheres. They observed the velocity variance increased with applied voltage (Locke et al. 2001). Buhai et al. (2008) studied EOF dispersion behavior in model porous media (VitraPor glass) closed to external flow and observed hydrodynamic backflow and the breaking of similitude between the electric and fluid fields. They also observed a transition from subdiffusive to superdiffusive dispersion as the electric field strength or pore size was increased.

In a porous medium completely closed to external flow, the electroosmotic and induced pressure-driven flows combine to yield zero net flow and maximum electroosmotic pressure. Electroosmotic permeability, or the resistance to electroosmotic flow, is independent of the pore size. An applied electric field generates the same electroosmotic slip velocity in capillaries of different diameter (O'Brien 1986). However, the resistance to pressure-induced reverse flow, hydraulic permeability, depends on the pore surface-to-volume ratio and decreases with the square of the pore size. This is balanced by the electroosmotic pressure, which scales inversely with the pore size squared and hence inversely with the hydraulic permeability (Adamson and Gast 1997; Bear 2013).

In the case of an unconsolidated porous medium, electroosmotic pressure can be sufficient to induce fluidization or consolidation. For particle classes subject to fluidization, the theoretical minimum fluidization velocity $u_{\mathrm{mf}}$ is proportional to the square of the particle diameter (Richardson and Harker 2002). As pressure is increased, rearrangement of the solid matrix typically occurs before actual fluidization. With the onset of fluidization, dispersion scales strongly with fluid velocity (Chung and Wen 1968).

Highly cohesive particles may resist fluidization, but strong interparticle attraction is associated with channel and cavity formation during packing and such defects are associated with greater dispersion (Schure and Maier 2006). Massimilla and Donsi (1976) compared the magnitude of van der Waals and gravitational forces acting on particles and found a crossover near $100 \mu \mathrm{m}$ (Massimilla and Donsi 1976). Their results are consistent with other experimental work indicating difficulty in fluidizing beds below about $40 \mu \mathrm{m}$. As the particle size is decreased, channels become more stable and the bed more difficult to fluidize (Baerns 1966). Electroosmotic pressure induces shear flow in channels and cavities, which enhances dispersion and may induce compaction or consolidation depending on the compressibility of the matrix. Simulations indicate that hydrodynamic shear flows introduce irreversible compaction in colloidal suspensions (Seto et al. 2012).

The present experiments and simulations involve electroosmotic flows with HelmholtzSmoluchowski slip velocities $v_{\mathrm{S}}$ (1) on the same order as $\sqrt{6 D_{m} t} / t$, i.e., the diffusion distance over the experimental time, and the average pore velocity $\bar{v}<v_{s}$ in general. The sphere diameters and average pore velocities in the experiments correspond to the Peclet number range, $0 \leq P e \equiv \bar{v} d / D_{m} \leq 25$, which spans from diffusion-dominated transport to mechanical dispersion. In the diffusion-dominated regime, $P e<1$, one expects restricted diffusion, where asymptotic $D \approx D_{m} / \tau$, with $\tau$ the tortuosity, implying that $D(t)$ is a decreasing function of time because $\tau>1\left(\tau=L_{e}^{2} / L^{2}\right.$, where $L$ is the length of the domain and $L_{e}$ the average length of current flow (Bear 2013). In the mechanical regime, roughly $P e>10$, dispersion is well predicted by a power-law relationship of the form $D_{L} / D_{m}=\alpha P e^{\beta}$, where $\beta>1$, implying $D_{L}(t)$ is an increasing function of time. The present experiments 
and simulations investigate $D(t)$ in the pre-asymptotic regime. Where $D(t)<D_{m}$, the preasymptotic behavior is subdiffusive, while in cases where $D(t)>D_{m}$, the pre-asymptotic behavior is superdiffusive. The timescale for asymptotic dispersion depends on the transport mechanism. Under PDF, diffusion is the mechanism for exchange in dead-end pores and the no-slip boundary layer. Where the characteristic length scales can be expressed as an equivalent particle diameter, the dependence is often assumed to be $t \propto d^{2} / D_{m}$. Under EOF, the exchange mechanism includes convection due to the slip velocity (balanced by backflow in dead-end pores) in addition to diffusion. $D(t)$ is a time-dependent tensor (Bear 2013), and where isotropy is not assumed, longitudinal or axial dispersion is denoted as $D_{L}$ and refers to the $D_{z z}$ component of $D$. Transverse dispersion is denoted by $D_{T}$ and refers to $D_{x x}$ and $D_{y y}$, under the assumption of transverse isotropy. In the case of no flow, the dispersion coefficient differs from the effective diffusivity in porous media as, $D_{\text {eff }}=\varepsilon D(t)=\varepsilon \theta D_{m} / \tau$, where $\varepsilon$ is the porosity and the constrictivity, $\theta$, is assumed equal to one in the present work. The symbol $D_{0} \equiv D_{m}$ is used in the figures for clarity.

The constitutive equation describing current flow in porous media is $J=\sigma_{\text {eff }} E$, where $J$ is the current density with unit $\mathrm{A} / \mathrm{m}^{2}, \sigma_{\text {eff }}$ is the effective conductivity with unit $\mathrm{S} / \mathrm{m}$, and $E$ is the macroscopic or applied electric field with unit $\mathrm{V} / \mathrm{m}$. The current density is approximated as $J=I / A$, where $A$ is the cross-sectional area of the porous medium perpendicular to the orientation of the applied field, and the current $I=\sigma_{f} V_{f}$ from Ohm's Law, with $\sigma_{f}$ the fluid conductivity and $V_{f}$ the flux with unit $\mathrm{V}$ m.

The Helmholtz-Smoluchowski relation describes EOF at a mesoscopic length scale where the electric double layer (EDL) thickness is much smaller than the pore size. It predicts the apparent slip velocity along a pore surface,

$$
v_{s}=\mu_{0} E=\frac{\epsilon_{0} \epsilon_{r} \zeta}{\mu} E
$$

where $\mu_{0}$ is the electrophoretic mobility, $\epsilon_{0}$ and $\epsilon_{r}$ are the absolute and relative permittivity, $\mu$ the dynamic viscosity, $\zeta$ the surface potential, and $E$ the local electric field. Note, $E$ is used to denote both the applied and the local electric field and the meaning depends on the context (Probstein 1989). The values $\epsilon_{0}=8.854 \times 10^{-12} \mathrm{~F} / \mathrm{m}, \epsilon_{r}=75.0$, and $\mu=0.001 \mathrm{~Pa}$ s are assumed in all cases.

The constitutive equation describing electroosmotic flow through porous media is a generalization of Eq. (1), $Q / A=K_{e} E$, where $Q$ is the volumetric flow rate with unit $\mathrm{m}^{3} / \mathrm{s}$ and $K_{e}$ is the electroosmotic permeability with unit $\mathrm{m}^{2} /(\mathrm{V} \mathrm{s})$, defined as $K_{e} \equiv \mu_{0} \sigma_{\text {eff }} / \sigma_{f} \equiv \mu_{0} \varepsilon / \tau$.

The electroosmotic pressure can be defined in an equilibrium sense by expressing the volumetric flow as the sum of hydraulic (Darcy's Law) and electroosmotic contributions. In the absence of an external applied pressure gradient,

$$
Q / A=-\frac{K_{h}}{\mu} \nabla p+K_{e} E
$$

where $K_{h}$ is the hydraulic permeability with unit $\mathrm{m}^{2}$ and $\nabla p$ the pressure gradient (Casagrande 1952). It follows that $\nabla p=0$ when $Q / A=v_{s}$ and $K_{e}=\mu_{0}$ (i.e., an open tube), and $\nabla p=\nabla p_{\max }=\left(\mu K_{e} / K_{h}\right) / E$ when $Q / A=0$ (i.e., a closed porous medium). 


\section{Methods}

\subsection{Dynamic NMR Transport Measurement}

NMR measurements of hydrodynamic dispersion induced by electroosmosis were obtained in a porous medium constructed from glass spheres. The sample cylinder was closed to external flow, and an internal flow was induced by an applied electric field using a Keithly 2410 SourceMeter. NMR measurements were obtained on a DRX250 Bruker spectrometer with a 10-mm-diameter radiofrequency (RF) coil and a 1.4-T/m, three-dimensional microimaging gradient probe. All experiments were conducted at a constant temperature of $20^{\circ} \mathrm{C}$.

The signal acquired in an NMR experiment may be sensitized to measure translational motion over a range of displacements from $10^{-8}$ to $10^{-3} \mathrm{~m}$ on timescales from $10^{-4} \mathrm{~s}$ to $1 \mathrm{~s}$ (Callaghan 1991; Fukushima 1999). This measurement of translational motion, whether via self-diffusion, coherent velocity or dispersion, is made by the strategic application of a pair of rectangular narrow gradient pulses of amplitude $g$, duration $\delta$ and separation $\Delta$, termed the pulsed gradient spin echo sequence, which encodes for displacements. Pulsed gradient NMR enables the measurement of the time-dependent effective diffusion coefficient $D(t)$ in complex systems.

In the NMR experiments presented in this paper, a variant of the pulsed gradient experiment using a stimulated echo sequence (PGSTE) was combined with an electrophoretic pulse as shown in Fig. 1 to allow for motion encoding while electroosmotic transport is being induced. This electrophoretic nuclear magnetic resonance (eNMR) technique was originally presented by Holz, Seiferling and Mao (Holz et al. 1993) and further developed by others (He et al. 1999; Holz et al. 2001; Stilbs and Furo 2006).

\subsection{NMR Measurement in Sphere Packings}

The sphere packing sample holder was a polyether ether ketone (PEEK) cylinder (ID $=6.55 \mathrm{~mm}, L=108 \mathrm{~mm}$ ) fitted with frits, platinum wire electrodes and PEEK plugs at both ends according to the recommendations in (Holz et al. 2001). Current was supplied through shielded wires. The length of the section between the two electrodes was $80 \mathrm{~mm}$. One end of the cylinder was fitted with a platinum electrode and frit to contain the spheres and sealed with teflon tape. In order to remove impurities, the glass beads were washed with $10 \%$

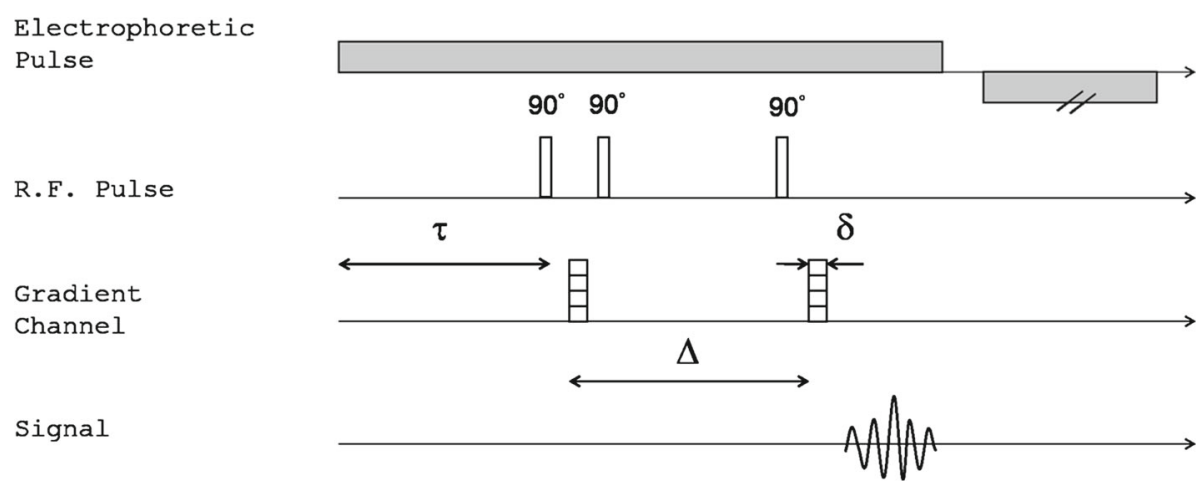

Fig. 1 PGSTE pulse sequence and electrophoretic pulse. $\tau$ is the time between initiation of the electrophoretic pulse and the first r.f. pulse, $\delta$ and $\Delta$ are the gradient pulse duration and separation, respectively. A second electrophoretic pulse of opposite sign and equal duration is applied after the signal is measured 


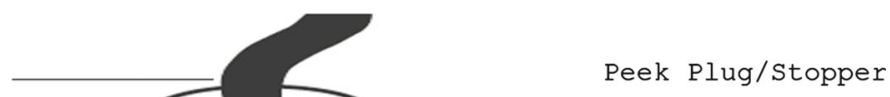

Platinum Wire Electrodes

Coaxial Cable

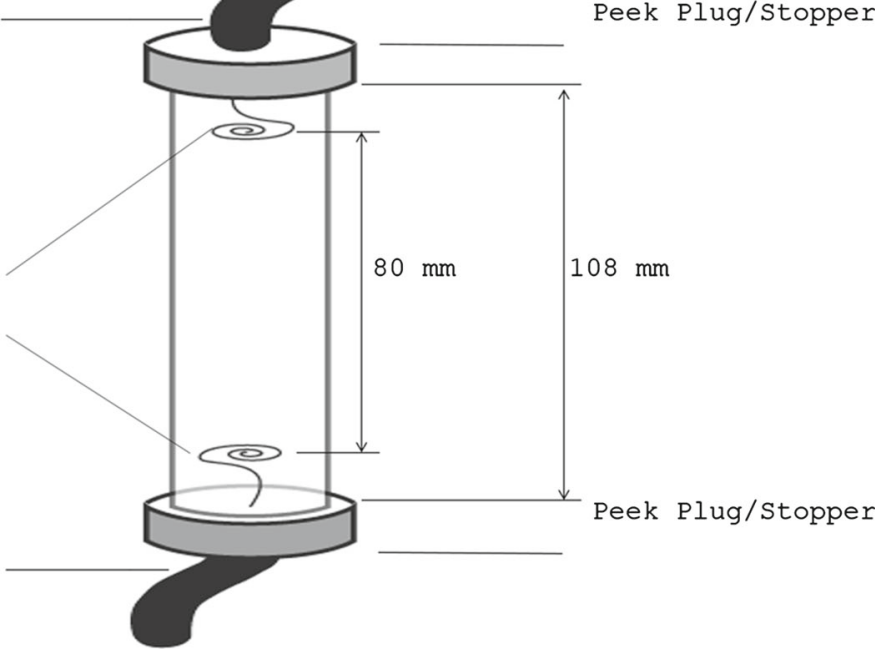

Fig. 2 Sample chamber for glass beads

$\mathrm{HCl}$ and rinsed with deionized water. The spheres were immersed in a $1 \mathrm{mM} \mathrm{KCl}$ solution and incrementally added into the cylinder while accumulated air bubbles were removed from the sphere packing by gentle stirring and cylinder vibration. In order to have a chemically stable, steady solution, sphere packs with fresh $1 \mathrm{mM} \mathrm{KCl}$ solution were rebuilt after every set of experiments that involved the presence of electroosmotic flow. Each set of experiments with an applied electric field included five NMR experiments that differ in their displacement encoding time $\Delta$. The second PEEK plug and electrode were then fitted, and the cylinder was sealed (Fig. 2). The electrodes were connected to a Keithly 2410 source meter, which was triggered by the NMR spectrometer to supply the necessary constant voltage pulses to the electrodes in step with the NMR sequence.

Borosilicate glass spheres of five different sizes, ranging in mean diameter from $30 \mu \mathrm{m}$ to $1000 \mu \mathrm{m}$, were obtained from Cospheric Microspheres. The zeta potential was measured for each size of glass spheres immersed in $1 \mathrm{mM} \mathrm{KCl}$ solution (Table 3) using a PALS Zeta Potential Analyzer (Ver. 5.59, Brookhaven Instruments Corp). The mean zeta potential value was obtained after 10 runs for each bead size. The zeta potential data are impacted by sedimentation of these large $(>10 \mu \mathrm{m})$ particles and are presented merely to demonstrate the values are of similar order of magnitude for all particles.

The gradient amplitude $g$ was varied from 0.006 to $0.12 \mathrm{~T} / \mathrm{m}$, using pulse duration $\delta=3 \mathrm{~ms}$ and pulse separation $\Delta$ varying from 50 to $400 \mathrm{~ms}$. The electrophoretic pulse was turned on $2.5 \mathrm{~s}$ before the initial RF pulse to allow the electroosmotic flow to reach steady state. After data acquisition, the applied voltage was inverted and a second electrophoretic pulse of identical duration was applied to reverse the effects of the electroosmotic flow and minimize gas bubble formation at the top electrode. Finally, an interval of $13 \mathrm{~s}$ was allowed between experiment repetitions to minimize Joule heating in the sample. The total time for one NMR diffusion coefficient measurement at a specific value of observation time $\Delta$ was $27 \mathrm{~min}$.

\subsection{Pore-Scale Simulation}

Electroosmotic flow and dispersion were simulated under the assumptions of a thin electric double layer (EDL), uniform surface charge, steady flow and a conservative, charge-free 
Table 1 Simulated and experimental sphere packings

Sample type, dimensions and porosity. Simulated packing dimensions given in terms of particle diameter

\begin{tabular}{llll}
\hline & Type & Dimensions & $\varepsilon$ \\
\hline Sim. & Bulk & $4.78 \mathrm{~d} \times 4.78 \mathrm{~d} \times 4.78 \mathrm{~d}$ & 0.40 \\
Sim. & Bulk & $9.76 \mathrm{~d} \times 9.76 \mathrm{~d} \times 9.76 \mathrm{~d}$ & 0.36 \\
Sim. & Cyl. & ID $=6.55 \mathrm{~d} \times \mathrm{L}=79.6 \mathrm{~d}$ & 0.42 \\
Sim. & Cyl. & ID $=6.55 \mathrm{~d} \times \mathrm{L}=9.77 \mathrm{~d}$ & 0.43 \\
Exp. & Cyl. & ID $=6.55 \mathrm{~mm} \times \mathrm{L}=80 \mathrm{~mm}$ & est. 0.42 \\
\hline
\end{tabular}

solute in a porous medium saturated with a neutral electrolyte (Probstein 1989). The spatial resolution of the pores was on the order of one micron or larger, several orders of magnitude larger than the EDL. The zeta potential was assumed to be uniform over all solid surfaces. This assumption is based on the small EDL thickness relative to the pore size, the washing of the particles and replacement of the ionic solution between each sample run, and the use of an opposite charge electric field pulse in each increment of the eNMR (Fig. 1) experiment to redistribute the solution ions. The electric field was calculated under the assumption of a neutral fluid electrolyte and non-conducting solid surfaces in the porous matrix and confining walls. The EDL slipping plane velocity (1) was applied at all solid surfaces using the computed electric field and the zeta potential values from Table 3 (Probstein 1989). The applied voltage drop was constant in time (DC), and the fluid flow field was assumed to have a steady, time-independent solution. Solute transport was governed by hydrodynamic forces only, and electrophoretic motion was not considered.

\subsubsection{Simulated Porous Media}

Sphere packings were generated with a hard-sphere molecular dynamics algorithm which slowly compresses a dilute array of spheres to a specified porosity (Maier et al. 2000). Packings were created in a cylinder to study wall effects associated with relatively large sphere-to-cylinder diameter ratios, while periodic sphere packings were created to approximate the "bulk" packing structure which predominates in columns with smaller ratios. Planar boundaries were imposed to simulate packings closed to external flows.

When computing the electric, velocity and effective diffusion fields, the sphere packings were mapped to 3D regular grids. Grid cells were classified as solid or void voxels, yielding a "stair-step" representation of solid surfaces. Packing and sample dimensions are summarized in Table 1.

\subsubsection{Simulated Electrical Field}

An electric field was simulated under the assumptions of a neutral electrolyte with conductivity $\sigma_{f}$, a non-conducting solid matrix with uniform surface potential, and fixed potential external boundaries. Under these assumptions, the potential field is described by Laplace's equation, $\nabla^{2} \phi=0$ subject to no-flux boundary conditions at the solid surfaces (Probstein 1989).

The potential field was approximated with a lattice-Boltzmann (LB) method, which solves the discrete-velocity Boltzmann equation for the charge and current density distributions. The LB method with single-time relaxation (BGK) applies a finite-difference discretization to the Boltzmann equation (Sterling and Chen 1996). Cartesian space is discretized on a cubic lattice and velocity space is discretized by $q=7$ lattice vectors, $\mathbf{e}_{i}, i=1, \ldots, q$, consisting of the 
three Cartesian unit vectors, their negations and the null vector (the D3Q7 LB-BGK method). In dimensionless form,

$$
f_{i}\left(\boldsymbol{x}+\boldsymbol{e}_{\boldsymbol{i}}, t+1\right)=f_{i}(\boldsymbol{x}, t)-\frac{1}{\omega}\left[f_{i}(\boldsymbol{x}, t)+f_{i}^{E Q}(\rho, \boldsymbol{v})\right]+\mathrm{F}(\boldsymbol{x}) \cdot \boldsymbol{e}_{\boldsymbol{i}}, i=1, \ldots, q
$$

where $f_{i}$ denotes the charge carrier distribution function associated with $\mathbf{e}_{\mathbf{i}}$, and the position $\boldsymbol{x}$ denotes the center of the lattice cell. The dimensionless charge and current densities are defined as the velocity moments

$$
\rho=\sum_{i=1}^{q} f_{i}, \rho \boldsymbol{v}=\sum_{i=1}^{q} f_{i} \boldsymbol{e}_{\mathbf{i}}
$$

where $v$ is the local drift velocity. The current density is related to the potential gradient through the conductivity, $\sigma_{f} \nabla^{2} \phi=\rho v$. The equilibrium distribution function, $f_{i}^{E Q}$, is a first-order expansion in the velocity, $f_{i}^{E Q}(\rho, v)=\rho\left[A_{i}+B_{i} e_{i} \cdot v\right]$, whose coefficients are derived to recover Poisson's equation (see, e.g., Wolf-Gladrow 2000). The no-flux condition is enforced at solid surfaces and periodic boundary conditions are used elsewhere. At a solid surface, where $\boldsymbol{x}$ is in a solid cell and $\boldsymbol{x}+\boldsymbol{e}_{\boldsymbol{i}}$ is in a neighboring fluid cell, $f_{i}(\boldsymbol{x}, t)$ is undefined and is therefore approximated as $f_{i}(\boldsymbol{x}, t)=f_{j}\left(\boldsymbol{x}+\boldsymbol{e}_{\mathbf{i}}, t\right)$, where $\boldsymbol{e}_{\mathbf{j}}=-\boldsymbol{e}_{\mathbf{i}}$, also known as the bounce-back technique. The fixed potential boundary values, $\phi_{0}$ and $\phi_{L}$ were assigned at the $z=0$ and $z=L$ planes of the numerical grid, corresponding to the ends of the cylinder or opposite faces of the box. Physical units are recovered from the dimensionless equation by assigning the lattice cell size $\Delta x$ and the ratio of lattice to physical charge densities.

The electrical flux in the axial direction is calculated by summing the $z$-component of the potential gradient. Let $u_{i}=\nabla_{z} \phi\left(x_{i}\right)$ denote the $z$-component of the potential gradient in the $i$ th grid cell with unit $\mathrm{V} / \mathrm{m}$. The mesoscopic expression for electrical flux through the $i$ th cell of the simulation grid is $(\Delta x)^{2} u_{i}$, where $\Delta x$ is the grid cell size. Integrating over the entire domain (flow through a volume) and dividing by the domain length give the total flux $V_{f}=(\Delta x)^{2} \sum_{i=1}^{n} u_{i} / L$. The dimensionless ratio of effective conductivity vs. fluid conductivity is solved from $V_{f} / A=\left(\sigma_{\text {eff }} / \sigma_{f}\right) E$, where the applied field is approximated as $E=\left(\phi_{0}-\phi_{L}\right) / L$, and $\phi_{0}, \phi_{L}$ denote fixed potential boundary values at $z=0$ and $z=L$.

\subsubsection{Simulation of Fluid Flow}

Fluid flow was simulated under the assumptions of steady, saturated flow of a neutral electrolyte at small Mach number in the thin-EDL limit. Fluid motion in the pore space is described by the incompressible Navier-Stokes equations, $\rho[\partial \boldsymbol{v} / \partial t+(\boldsymbol{v} \cdot \nabla) \boldsymbol{v}]=\rho F-\nabla p+\mu \nabla^{2} \boldsymbol{v}$, with appropriate initial and boundary conditions, where $v$ denotes the pore velocity, $\mu$ the dynamic viscosity, $F$ a body force and $p$ the pressure.

The equations of fluid flow were solved on a regular grid with the D3Q19 LB-BGK method (Qian et al. 1992). The method is given by (3), but in this case the $f_{i}$ denote fluid mass distribution functions, and the moments (4) denote the fluid mass and momentum densities. The equilibrium distribution function, in this case, is a second-order expansion in the velocity, whose coefficients are chosen to recover Navier-Stokes behavior in the lowMach-number limit (see, e.g., Chen et al. 1992). The dimensionless kinematic viscosity is given by $v_{l}=(2 \omega-1) / 6$ and the equation of state by $p=c_{s} \rho$, where $c_{s}^{2}=1 / 3$ is the square of the lattice sound speed. Physical units are recovered from the dimensionless equation based on these dimensionless lattice parameters, their equivalent physical definitions and the value of $\Delta x$. 
Flow was driven either by prescribed-velocity boundary conditions at the solid surfaces, corresponding to the Helmholtz-Smoluchowski slip velocity, or by a uniform pressure gradient in the case of non-electroosmotic, pressure-driven flows. In the latter case, the pressure gradient was implemented as a uniform body force, $F$ in (3), with no-slip conditions at all solid boundaries implemented by bounce-back, described in the previous section. Gravitational force was not simulated. For geometries with open external boundaries, periodic boundary conditions were used to wrap the flow around to the opposite face of the simulation box.

The flow simulation was initiated at zero velocity and iterated to a steady state, i.e., until the relative change in the mean pore velocity was less than a specified tolerance

$$
\text { tol }>\eta=2\left|\bar{v}^{k+m}-\bar{v}^{k}\right| / \mid \bar{v}^{k+m}+\bar{v}^{k}
$$

where the superscript $k$ refers to the $k$ th iteration and $m$ is a small integer that filters fluctuations in $\eta$. The values $m=10$ and tol $=1 \times 10^{-6}$ were used unless otherwise noted.

The volumetric flow rate, $Q$, is calculated by summing the $z$-component of the fluid velocity, similar to the case of electrical flux described earlier. Let $v_{i}=v\left(x_{i}\right)$ denote the axial velocity component in the $i$ th grid cell. Integrating $(\Delta x)^{3} v_{i}$ over the entire domain and dividing by the domain length give the volumetric flow $Q=(\Delta x)^{3} \sum_{i=1}^{n} v_{i} / L$ with unit $\mathrm{m}^{3} / \mathrm{s}$.

\subsubsection{Velocity Distribution Functions}

The results of flow simulations are presented as empirical velocity distribution functions. Let $H_{j}$ denote the velocity histogram

$$
H_{j}=\sum_{i=1}^{n} \mathbf{1} v_{j}^{h}-h<v_{i}<v_{j}^{h}+h \quad, \quad j=1, \ldots, M
$$

where the indicator function 1 counts the grid cell velocities falling into $M$ bins of width $2 h=\left(v_{\max }-v_{\min }\right) / M$ and $v_{j}^{h}=v_{\min }+2(j-1 / 2) h$ are the bin midpoints. The grid cell velocity $v_{i}$ maps to the $j$ th bin as $\left.j=\min \left(M,\left\lfloor v_{i}-v_{\min }\right) / 2 h\right\rfloor+1\right)$. The discrete probability density function is defined as $f \quad v_{j}^{h}=H_{j} /(2 h n)$, such that $2 h \sum_{j=1}^{M} f\left(v_{j}\right)=1$, which enables the comparison of distributions with different ranges. If $v \mapsto j, f(v)$ is the scaled probability that $v$ falls into the $j$ th bin,

$$
\operatorname{Pr} v_{j}^{h}-h<v<v_{j}^{h}+h=2 h f(v) .
$$

\subsubsection{Simulation of Dispersion}

Solute transport was simulated in the digital geometry under the assumptions of a dilute, conservative, charge-free solute moving only under the influence of Brownian motion and solvent advective velocity. Transport was modeled by the motion of tracer particles governed by the Langevin equation (Maier et al. 2003). The connection between the convection diffusion equation (CDE) and the Langevin equation is described by Brenner (Brenner and Edwards 1993). The Langevin equation for the position of an individual tracer was solved by the forward Euler method,

$$
\boldsymbol{x}(t+\Delta t)=\boldsymbol{x}(t)+\boldsymbol{v}(\boldsymbol{x}(t)) \Delta t+\xi \overline{2 D_{m} \Delta t}
$$


where $\boldsymbol{x}$ is the position vector, $\boldsymbol{v}(\boldsymbol{x})$ is interpolated from LB velocity field, and $\xi$ is a random vector on the surface of a sphere of radius $\sqrt{3}$ (see, e.g., Kloeden 1992). Solute dispersion was calculated as the time rate of change in the particle displacement variance, $D(t)=$ $(1 / 2) \mathrm{d} \boldsymbol{\sigma} / \mathrm{d} t$, where $\boldsymbol{\sigma}(t)=(1 / n) \sum_{i=1}^{n}\left(\boldsymbol{x}_{\boldsymbol{i}}-\overline{\boldsymbol{x}}\right)\left(\boldsymbol{x}_{i}-\overline{\boldsymbol{x}}\right)^{T}$ and $n$ denotes the number of particles.

\section{Results}

\subsection{Electroosmotic Flow in a Closed Fluid-Filled Cylinder}

Electroosmosis in a closed cylinder with current flow along the axis is characterized by flow along the walls, in the direction of the applied electric field, and a counterflow in the center, which combine to yield zero net flow (Manz et al. 1995; Rice and Whitehead 1965; Yao and Santiago 2003). The flow equation is a superposition of electroosmotic flow and a reverse pressure-driven flow. The electroosmotic flow along the wall induces the reverse pressure gradient, which is proportional to the applied electric field (Marcos et al. 2005). Electroosmotic flow in the thin-EDL limit was simulated to illustrate the phenomenon of pressure-driven reverse flow. Figure 3 shows contours of the axial velocity in a cross section. The flow is upward along the wall, turns at the top and returns down the center of the cylinder. Most of the cylinder volume is occupied by slower return flow. The forward and reverse flows have the same mean velocity exactly at the midplane, but away from the midplane, the mean return velocity decreases. At the midplane, the computed velocity profile is approximately parabolic (not shown) and the transverse velocity component vanishes, $v_{x}=v_{y}=0$. The axial velocity also vanishes for radial coordinate $r_{0}=R \sqrt{2}$, which divides the midplane disk into two annular regions of equal area, i.e., $v_{z}\left(r_{0}\right)=0$. Figure 3 also shows the axial velocity distribution over the entire cylinder. The positive velocities have an approximately uniform distribution, indicating that the electroosmotic profile is fairly constant along the cylinder length. The negative velocities, however, are distributed in a roughly exponential form, which is the characteristic form (with opposite sign) of pressure-driven flows in packed spheres (Maier et al. 2000). In this respect, the velocity distribution in a closed cylinder resembles a porous medium more than an open cylinder.
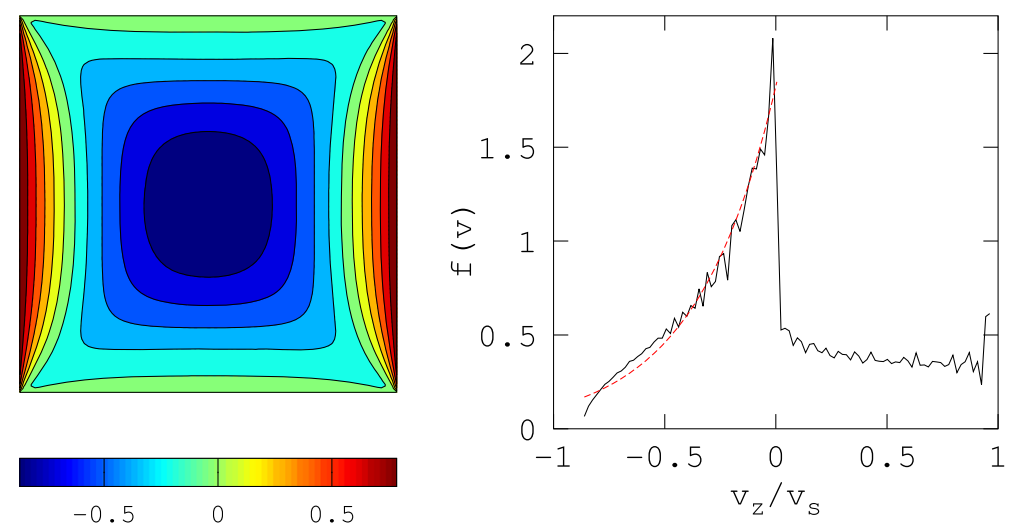

Fig. 3 Cross section of EOF in a closed cylinder. Contours of the scaled axial velocity $v_{z} / v_{S}$ are shown at left. Scaled axial velocity distribution with fitted function $f=\alpha e^{\beta v}$ are shown as dashed line, at right. The cylinder I.D. $=L=1 \mathrm{~mm}$, with $E=25 \mathrm{kV} / \mathrm{m}, \zeta=-0.03 \mathrm{~V}$, and $\Delta x=10^{-5} \mathrm{~m}$ 


\subsection{EOF and PDF Simulations in Open Sphere Packings}

Simulation results for EOF in open sphere packings have been presented by Hlushkou et al. $(2005,2007)$. Their results illustrate the shape of the velocity probability distribution function $f(v)$. Their results also demonstrate a linear relationship between the applied electric field strength and the mean pore velocity. They also note the variation of electroosmotic velocities within the pore spaces of the sphere packing, in contrast to uniform EOF flow in a simple tube.

In the present work, EOF and PDF were simulated in bulk packings with periodic flow boundaries applied in all three dimensions (Table 1). The applied potential gradient was $5 \mathrm{kV} / \mathrm{m}$ oriented along the $\mathrm{z}$ axis. The zeta potential was matched to the experimental sphere diameter according to Table 3. The PDF body force was chosen to match the EOF mean velocity. The grid resolution was $\Delta x=d / 40$ except where otherwise noted.

Figure 4 illustrates the effect of varying the sphere diameter and fluid driving force on the shape of the velocity distribution. The results show that for both EOF and PDF, the velocity distributions collapse when scaled by their mean. The scaled EOF velocity distribution is roughly Gaussian with mode approximately equal to its mean, i.e., $v / \bar{v} \approx 1$, and only a small fraction of velocities near zero. In contrast, the scaled PDF velocity distribution has its mode near $v=0$ and decreases exponentially. The relatively small fraction of zero velocities in EOF indicates most of the fluid is in motion. The scaled EOF and PDF modes have similar amplitude, but the EOF distribution is narrower, indicating lower velocity variance and consistent with the idea that EOF is more uniform. Note that the maximum EOF velocities attained in the packings were several times greater than the maximum imposed slip velocity $v_{S}$ tabulated in Table 2, whereas the mean velocities are roughly half the value of $v_{S}$. The actual volume of fluid moving faster than $v_{S}$ is small but clearly visible in Fig. 4 . The shape of the EOF distributions contrasts with the experimental results in (Locke et al. 2001, Fig. 7), where the EOF distributions more closely resembled a PDF with a mode at $v=0$ and decreasing exponential form. The difference results from the orientation of the electrical field with respect to the gravitational field. Locke et al. (2001) placed the cathode at the top of the column, so EOF was against gravity, while the present experiments placed the cathode at the bottom.

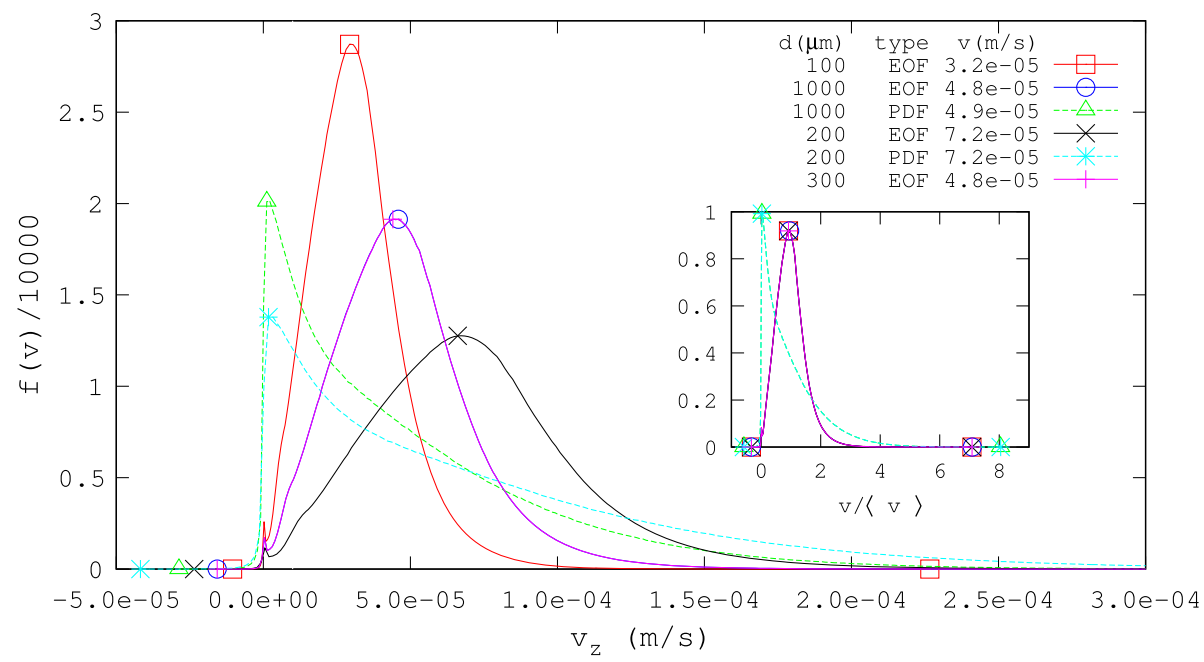

Fig. 4 Velocity histograms for EOF and PDF simulated in an open sphere packings for different values of sphere diameter and driving force. The value of the mean pore velocity $\bar{v}$ is shown in the legend. The curves for EOF and PDF collapse when scaled by $\bar{v}$ (inset) 
Table 2 Velocity statistics for open sphere packings, including flow type, sphere diameter $d$, surface potential $\zeta$, slip velocity $v_{S}$ from (1), and the minimum, maximum, mean and standard deviation of the simulated velocity field

\begin{tabular}{llllllll}
\hline Flow & $d(\mathrm{~mm})$ & $\zeta(\mathrm{V})$ & $v_{\mathrm{S}}$ & $v_{\min }$ & $v_{\max }$ & $\bar{v}$ & $\mathrm{~s}(v)$ \\
\hline EOF & 100 & -0.02 & $-6.72 \mathrm{e}-5$ & $-1.08 \mathrm{e}-5$ & $2.27 \mathrm{e}-4$ & $3.20 \mathrm{e}-5$ & $1.57 \mathrm{e}-5$ \\
EOF & 200 & -0.045 & $-1.48 \mathrm{e}-4$ & $-2.42 \mathrm{e}-5$ & $5.11 \mathrm{e}-4$ & $7.20 \mathrm{e}-5$ & $3.54 \mathrm{e}-5$ \\
PDF & 200 & & & $-4.26 \mathrm{e}-5$ & $5.80 \mathrm{e}-4$ & $7.19 \mathrm{e}-5$ & $6.72 \mathrm{e}-5$ \\
EOF & 300 & -0.03 & $-1.00 \mathrm{e}-4$ & $-1.61 \mathrm{e}-5$ & $3.41 \mathrm{e}-4$ & $4.80 \mathrm{e}-5$ & $2.36 \mathrm{e}-5$ \\
EOF & 1000 & -0.03 & $-1.00 \mathrm{e}-4$ & $-1.61 \mathrm{e}-5$ & $3.41 \mathrm{e}-4$ & $4.80 \mathrm{e}-5$ & $2.36 \mathrm{e}-5$ \\
PDF & 1000 & & & $-2.92 \mathrm{e}-5$ & $3.97 \mathrm{e}-4$ & $4.93 \mathrm{e}-5$ & $4.61 \mathrm{e}-5$
\end{tabular}

Units are m/s unless otherwise noted. Applied field strength was $5 \mathrm{kV} / \mathrm{m}$

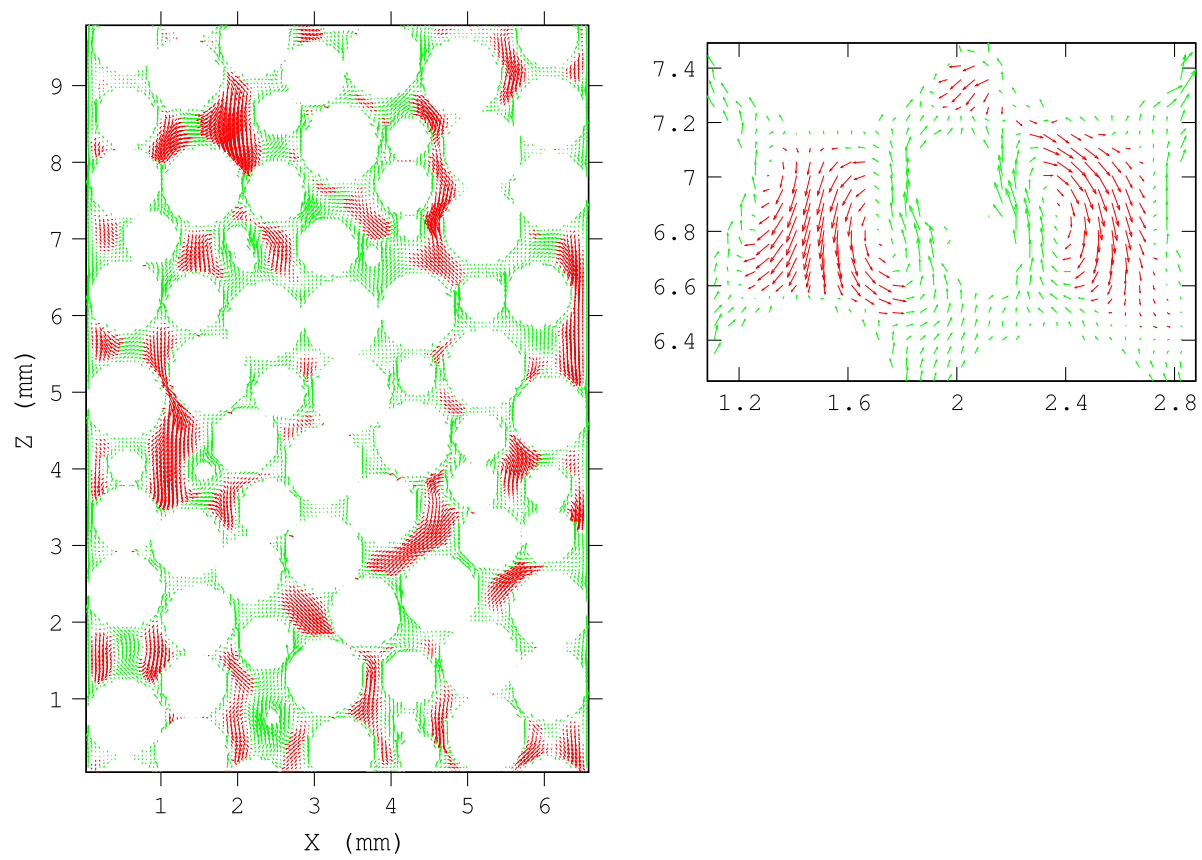

Fig. 5 EOF in a closed column packed with $1000 \mu \mathrm{m}$ spheres. Direction of potential flow is upward. Vector length corresponds to the velocity magnitude and vectors with a negative, or backflow, component are shown in $r e d$. Pore-scale rotational flow shown in the enlargement at right

\subsection{EOF and PDF Simulations in Closed Sphere Packings}

EOF was simulated in cylindrical and bulk packings closed to external flow (Table 1), using a $5 \mathrm{kV} / \mathrm{m}$ applied potential gradient and zeta potential matched to sphere diameter (Table $3)$. Figure 5 illustrates flow patterns in a cross section of the closed cylindrical packing. The applied electric field is oriented in the vertical direction, and the return flow is downward (the force of gravity is not simulated). Axial and transverse velocity components have similar magnitudes. Slip flow appears everywhere at the sphere surfaces, while backflow appears concentrated in some pores and absent in others. The forward and reverse flow networks 

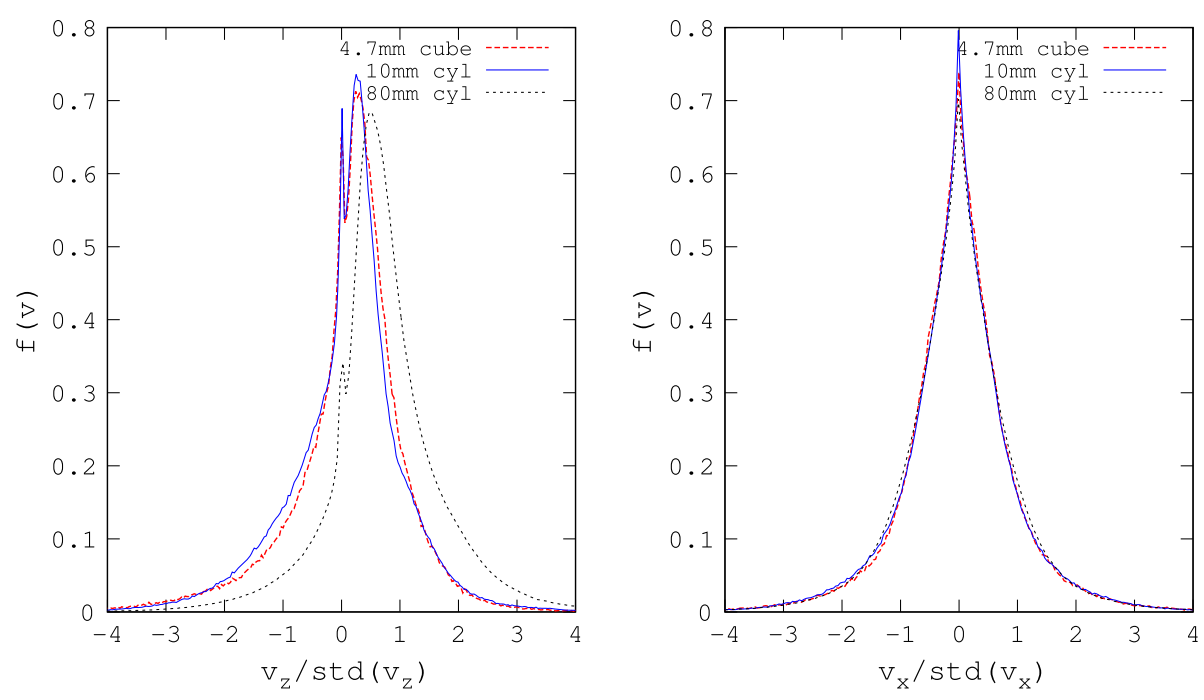

Fig. 6 Normalized axial (left) and transverse (right) velocity distributions in closed sphere packings. The 80-mm cylinder distribution illustrates the effect of simulation continuity errors

are not isomorphic to the pore network. Turning flows are common, and some pore-scale recirculation patterns are evident. Although recirculation cells are not uncommon, the flow patterns responsible for most of the recirculation flow have a longer length scale.

Axial velocity distributions exhibit symmetry about zero which is expected from continuity (Fig. 6), but the positive and negative tails differ in a way that reflects two different driving forces. The negative tail $(v / s(v)<-1)$ represents the pressure-induced reverse flow and the positive tail $(v / s>1)$ corresponds to the forward electroosmotic flow. The pressure-driven tail is better fit by an exponential distribution, while the electroosmotic tail is better fit by a Gaussian. The pressure-driven flow has a larger variance than the electroosmotic flow, which is also the case for PDF and EOF in open packings. These results are consistent with the observations in Locke et al. (2001) (Fig. 5) for $E=50 \mathrm{~V}$, where the electroosmotic tail (negative velocities in their case) appears less dense than the pressure-driven tail. The mean backflow pore and superficial velocities $v_{B}$ and $u_{B}$ are tabulated in Table 3. It was conjectured that the forward flow distribution might correspond to a pure electroosmotic flow, while the reverse flow distribution might conform to a pure pressure-driven flow, and some numerical fitting was performed to test this idea. EOF and PDF distributions from the open packs in Fig. 4 were superposed and compared to the closed pack in Fig. 6. The overall match was not very compelling, which confirms that electroosmotic pressure affects both forward and reverse velocities.

Transverse velocity distributions exhibit symmetry about zero and both tails conform more closely to an open EOF distribution than to PDF (Fig. 6). Their amplitudes and extreme values are similar to the axial velocity distributions, consistent with the similarity between transverse and axial velocity vector magnitudes captured in the spatially resolved Fig. 5. Closed and open velocity distributions are plotted together in Fig. 7. Closed and open packings with similar diameters have correspondingly similar modal amplitudes. The open packings have larger positive velocities, but the closed packings have a somewhat broader range and larger velocity variance.

Flow simulations in the closed sphere packings exhibited slower convergence to steady state and greater continuity errors than in open packings. Simulations in a cylindrical packing 
Table 3 Velocity statistics for closed sphere packings, including flow type, sphere diameter $d$, surface potential $\zeta$, slip velocity $v_{S}$ from (1), minimum fluidization velocity $u_{m f}$, and mean backflow pore and superficial velocities $v_{B}$ and $u_{B}$ from simulation

\begin{tabular}{llllll}
\hline$d(\mu \mathrm{m})$ & $\zeta(\mathrm{V})$ & $v_{\mathrm{S}}$ & $u_{\mathrm{mf}}$ & $v_{\mathrm{B}}$ & $u_{\mathrm{B}}$ \\
\hline 30 & -0.018 & $-6.08 \mathrm{E}-05$ & $-6.27 \mathrm{E}-06$ & $-1.80 \mathrm{E}-05$ & $-7.20 \mathrm{E}-06$ \\
50 & -0.017 & $-5.63 \mathrm{E}-05$ & $-1.74 \mathrm{E}-05$ & $-1.67 \mathrm{E}-05$ & $-6.67 \mathrm{E}-06$ \\
100 & -0.02 & $-6.72 \mathrm{E}-05$ & $-6.97 \mathrm{E}-05$ & $-1.99 \mathrm{E}-05$ & $-7.96 \mathrm{E}-06$ \\
200 & -0.045 & $-1.48 \mathrm{E}-04$ & $-2.79 \mathrm{E}-04$ & $-4.38 \mathrm{E}-05$ & $-1.75 \mathrm{E}-05$ \\
1000 & -0.03 & $-1.00 \mathrm{E}-04$ & $-6.97 \mathrm{E}-03$ & $-3.04 \mathrm{E}-05$ & $-1.22 \mathrm{E}-05$
\end{tabular}

Values of $50 \mu \mathrm{m}, 100 \mu \mathrm{m}$, and $200 \mu \mathrm{m}$ spheres are extrapolated from the results for $30 \mu \mathrm{m}$ based on the $\zeta$ potential. Applied field strength was $5 \mathrm{kV} / \mathrm{m}$. Units are $\mathrm{m} / \mathrm{s}$ unless otherwise noted

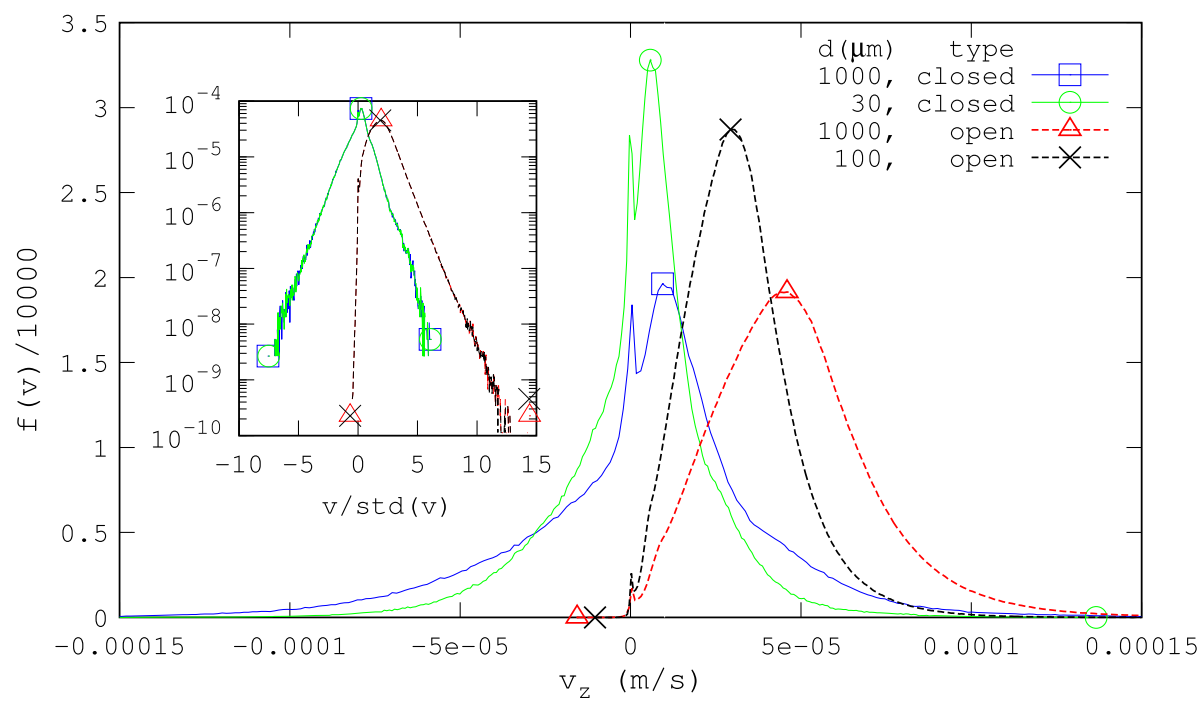

Fig. 7 EOF velocity distribution from a closed packed cylinder and an open $10 d \times 10 d \times 10 d$ periodic packing for different values of the sphere diameter and zeta potential ( $E=5 \mathrm{kV} / \mathrm{m}$ in all cases). The curves for open and closed packings collapse when scaled by the square root of the velocity variance (inset)

matching the experimental length $80 d$ converged slowly with $\eta=6 \times 10^{-5}$ after 50,000 iterations. The resulting partially converged velocity distribution is shown in Fig. 6. A shorter cylindrical packing with $L \approx 10 \mathrm{~d}$ matching the experimental diameter required on the order of 20,000 iterations for convergence and was used instead. But even with a shorter column, the number of LB iterations required to satisfy (5) was on the order of $10^{4}$, compared to $10^{3}$ for open packings. The average pore velocity should be zero, but the computed value of $\bar{v}$ exhibited the effects of continuity errors (see Fig. 6). For example, in the cylindrical closed pack, the computed value of $\bar{v}$ was approximately one order of magnitude smaller than $v_{S}$, at a resolution of $\Delta x=d / 20$. Although continuity errors depend upon the spatial resolution, it is the distance which pressure waves must travel that slows convergence to steady state in a closed packing. Recirculation patterns develop and pressure waves propagate on the length scale of the closed container, whereas in an open packing, downstream pressure fluctuations don't propagate far upstream. A conclusion is that implicit methods designed for steady-state 
solutions would be more efficient than the pseudo-transient method used here, for solving closed-pack flows.

The grid convergence rate indicates the dependence of error on spatial resolution. The calculated rate was approximately linear, indicating that the boundary condition implementation is restricting grid convergence, since the LB method is known to otherwise converge quadratically away from boundaries (Sterling and Chen 1996). Numerical investigations revealed the calculation of the electric field at the sphere surfaces as a source of error with poor grid convergence, even using the nominally second-order accurate boundary methods described in (Maier and Bernard 2010). Nonetheless, velocity distributions using spatial resolutions of $\Delta x=d / 20$ and $\Delta x=d / 40$ exhibit only incremental differences in amplitude and shape, indicating that qualitative features of the velocity field are captured by this level of resolution.

Flow curvature and shear gradients are much greater in a closed packing under EOF and place greater demands on the numerical methods. For example, recirculation cells require a minimum of around ten grid points to resolve the flow curvature using the present methods. Since the insphere diameter in pore spaces of a random monosize sphere packing ranges effectively from 0.15d to 0.40d (Mason 1971; Shiota and M. 1997) (6-16 grid points at a spatial resolution $\Delta x=d / 40$ ) only those recirculation cells on the order of the insphere diameter are resolved in the simulations.

\subsection{Dispersion Simulations in Open Sphere Packings}

Solute dispersion under pressure-driven flow has been simulated in sphere packings by a number of groups (Maier et al. 2000; Sullivan et al. 2005). Dispersion under electroosmotic flow has been presented in (Hlushkou et al. 2007). Their results demonstrate that for similar $P e$, dispersion is much greater under PDF, and they attribute this to the relative uniformity and lower variance of EOF. Dispersion under EOF and PDF in a model porous medium (VitraPor glass) open to flow was presented in Buhai et al. (2008). The short-time behavior ranged from subdiffusive to superdiffusive depending on the applied field strength and mean pore size. Although they did not indicate Peclet numbers, it is estimated that their results cover the range of $P e$ in the present work and extend somewhat further into the mechanical dispersion regime. Solute dispersion was simulated in open sphere packings under conditions of no flow, electroosmotic flow, and pressure-driven flow (Fig. 4). Tracer particle motion was simulated for a period of $1 \mathrm{~s}$, corresponding roughly to the NMR observation time.

\subsubsection{Restricted Diffusion}

The results obtained in the absence of the electric field and the absence of a pressure gradient demonstrate restricted diffusion, in which the dispersion coefficient is initially equal to bulk self-diffusion and decreases over time to a value theoretically equal to the tortuosity $\tau$ of packed spheres, which depends on the porosity. Previous simulations have found $\tau^{-1} \approx 0.74$ for loosely packed spheres (Ghassemi and Pak 2011; Maier et al. 2000) but a value in the range, $2 / 3<\tau^{-1}<1 / \sqrt{2}$ is often cited for closed packed spheres (Huizenga and Smith 1986). After one second of simulated time, the dispersion coefficients straddle this range. The explanation is in part that $D(t)$ was measured in the pre-asymptotic regime in most of the experiments. When diffusion dominates over convection, the time for asymptotic $D(t)$ is $t \propto d^{2} / D_{m}$, i.e., the time for solute to diffuse over the pore length scale. The value of $d^{2} / D_{m}$ is 500,45 , and $5 \mathrm{~s}$ for spheres of diameter 1000,300 , and $100 \mu \mathrm{m}$, respectively. Of these, only the smallest might be expected to be close to its asymptote while the larger diameter cases are certainly pre-asymptotic. The trends in $D(t)$ under no flow shown in Fig. 8 are 

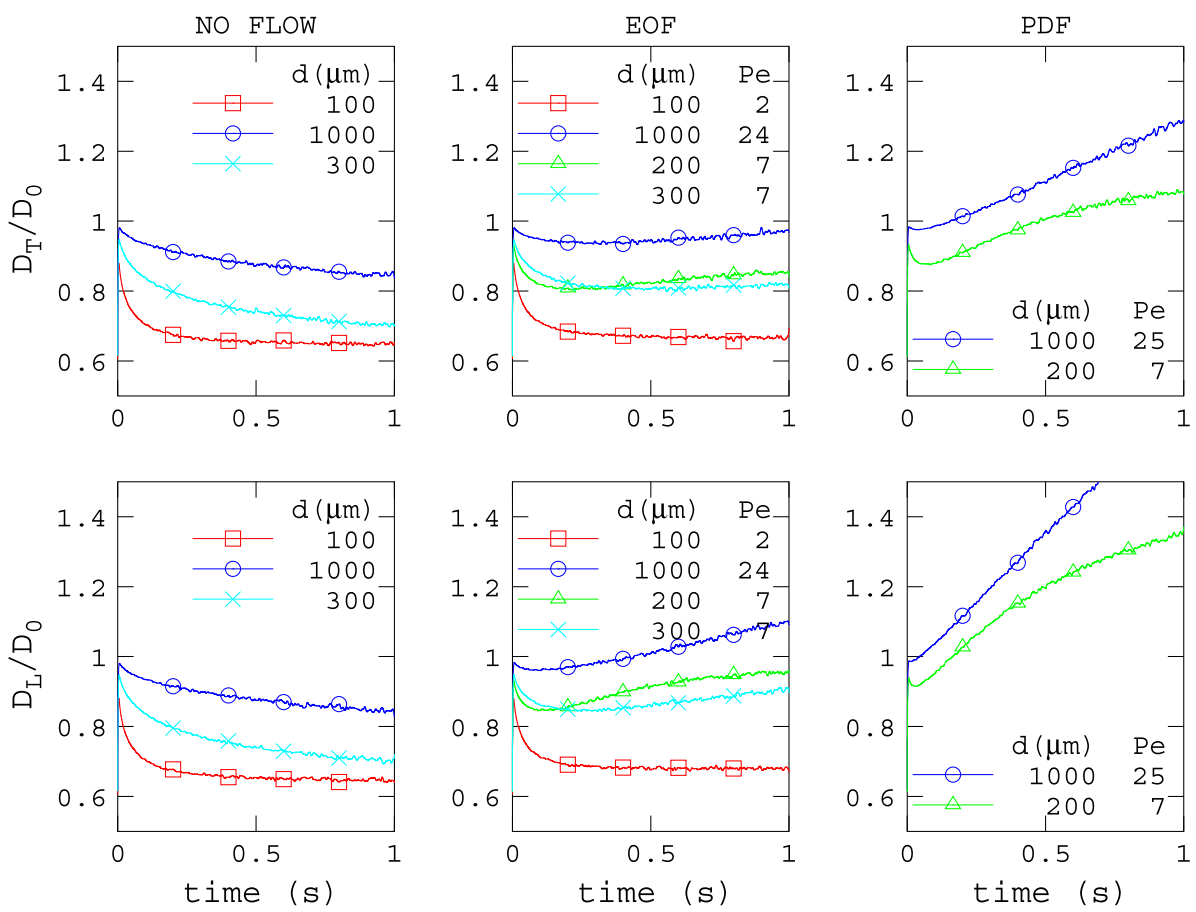

Fig. 8 Transverse and axial dispersion simulated in an open random sphere packing in the absence of the electric field and a pressure gradient and while undergoing EOF and PDF

consistent with these expectations. The coefficients for the larger spheres are still decreasing after one second of simulation. The fully developed value for $100 \mu \mathrm{m}$ is $D_{L} \approx 2 / 3$ which is consistent with some authors (e.g., Bear 2013).

\subsubsection{Dispersion Under EOF}

Based on the Peclet number range, $D_{L}$ should span the range from diffusion-dominated to mechanical-dominated dispersion. The $100-\mu \mathrm{m}$ packing has $P e=1.6$, and the value of $D_{L} / D_{m}$ is the same as that observed in experiments without electroosmotic flow and without a pressure gradient. The 200- and 300- $\mu$ m packings both have $P e=7$ and $D_{L} / D_{m}$ exhibits a transition from diffusion-dominated to mechanical-dominated dispersion. After one second, $D_{L} / D_{m} \approx 0.9$ in both packs, but the trend is upward (Note $P e=7.2$ for both cases because the values of $\zeta$ are -0.030 and $-0.045 \mathrm{~V}$ for 300 and $200 \mu \mathrm{m}$ spheres, respectively). The $1000 \mu \mathrm{m}$ sphere packing has $P e=24$ and $D_{L} / D_{m}=1.1$, and the trend is clearly upward.

\subsubsection{Dispersion Under PDF}

The results for PDF correspond to the mechanical-dispersion regime based on their magnitudes and upward trends. The (pre-asymptotic) values of $D_{L} / D_{m}$ are 1.3 and 1.1 for 1000 and $200 \mu \mathrm{m}$, respectively, after $1 \mathrm{~s}$. Previous PDF results (Maier et al. 2000) suggest asymptotic value of $D_{L} / D_{m} \approx 5$ for the $1000 \mu \mathrm{m}$ spheres $(P e=24)$ and $D_{L} / D_{m} \approx 2$ for the $200 \mu \mathrm{m}$ spheres $(P e=7)$. The magnitude of dispersion under PDF is greater than EOF, 

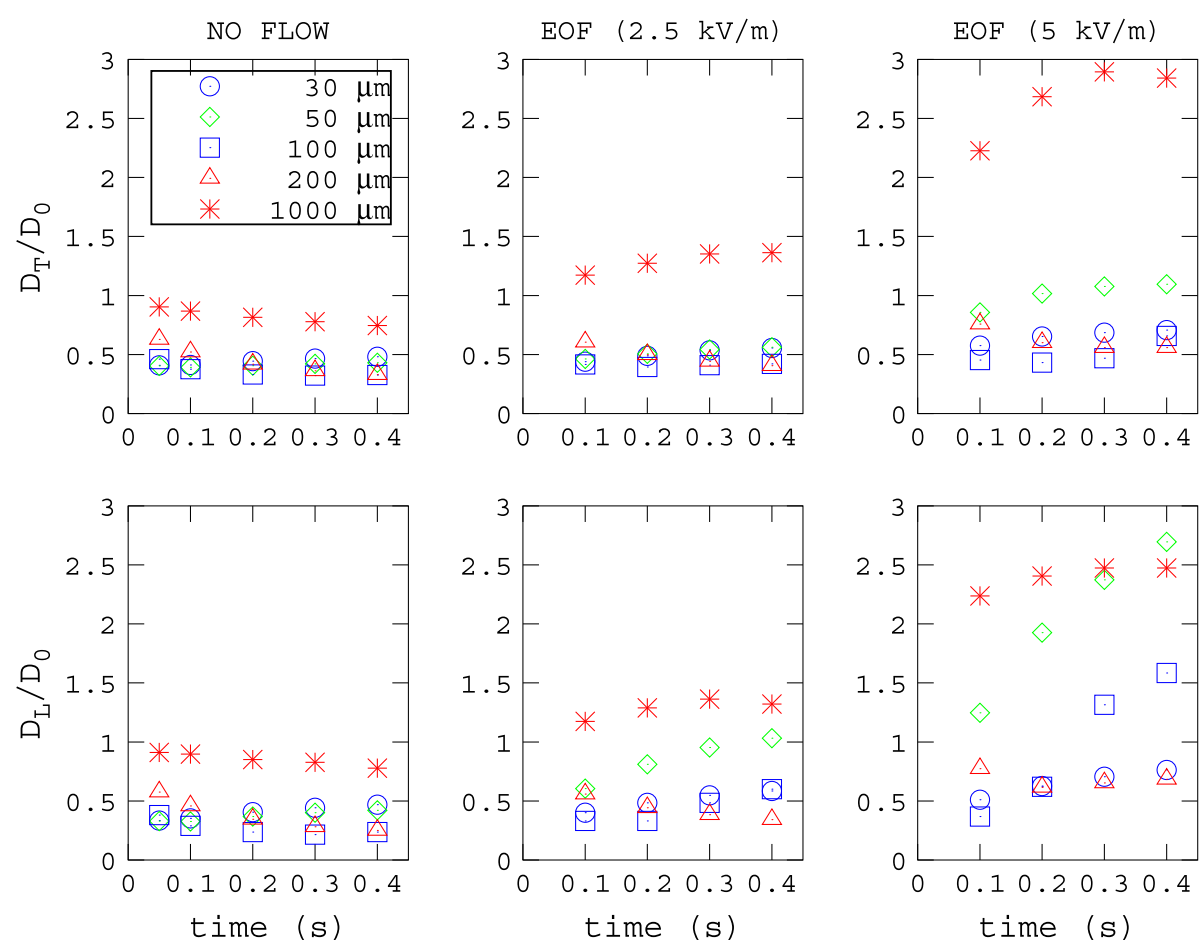

Fig. 9 Transverse and axial dispersion in closed sphere packings as a function of sphere diameter and applied field strength from NMR experiments

and the upward trend is stronger. This is consistent with the difference in velocity variance between the two flow types (see Table 2) and with the idea that dispersion is proportional to the velocity variance scaled by the mean, i.e., $\left.D_{L} \propto s^{2}\left(v_{z}\right) / \bar{v}\right)$ (Bear 2013).

\subsection{Dispersion Experiments and Simulation in Closed Sphere Packings}

Dispersion was measured in closed sphere packings for a range of sphere diameters using eNMR methods. The particle Peclet number for closed packings is defined as $P e=v_{B} d / D_{m}$, where $v_{B}$ is the mean backflow velocity. The values of $v_{B}$ are estimated from simulation and given in Table 3 along with the minimum fluidization velocity $u_{m f}=\left[\left(\rho_{s}-\rho_{f}\right) g \varepsilon^{3} d^{2}\right] /[150(1-\varepsilon) \mu]$ (Richardson and Harker 2002). $D_{T}$ and $D_{L}$ vs. time, $t$ are plotted in Fig. 9 for a case of absence of electroosmotic flow and for two values of the applied field gradient. Dispersion was isotropic in the transverse plane under EOF. Because the measurements were made over a maximum displacement time of $t=400 \mathrm{~ms}$, the presumption is that $D(t)$ is more fully developed for smaller $d$ unless evidence suggests heterogeneities that would extend the development time. Results for $D_{L}$ are compared with simulation and with the correlation (Edwards and Richards 1968)

$$
\frac{D_{L}}{D_{m}}=\frac{1}{\tau}+\frac{1}{2} P e
$$

which is expected to be an overestimate of EOF dispersion because it is based on PDF experiments. PDF has been found to be more dispersive than EOF in the present work as well as in (Hlushkou et al. 2007) and (Ghosal 2006). 


\subsubsection{Diffusion in the Absence of Electroosmotic Flow}

In the absence of flow, measurements indicate that dispersion is essentially isotropic and that $D(t)$ decreases with time to a value less than the molecular diffusion constant, for each sphere diameter (Fig. 9). The values of $D_{L}$ and $D_{T}$ differ by less than $5 \%$ for each sphere diameter, except for the 100 and $200 \mu \mathrm{m}$ spheres where, for reasons not entirely understood, $D_{L}$ is about $25 \%$ lower than $D_{T}$. In any case, it demonstrates the sensitivity of the restricted diffusion coefficient to the details of these small computer-generated packings. The values of $D_{L} / D_{m}$ and $D_{T} / D_{m}$ range from 0.25 to 0.5 at $t=0.4 \mathrm{~s}$ for all sphere diameters except for the $1000 \mu \mathrm{m}$ spheres, for which $D_{L} / D_{m} \approx D_{T} / D_{m}=0.75$ and the trend is decreasing.

\subsubsection{Dispersion Due to Electroosmotic Flow in Sphere Packs}

Under a $2.5-\mathrm{kV} / \mathrm{m}$ applied field in packed beds of spheres of $d=1000 \mu \mathrm{m}, P e=7.5$. Dispersion is isotropic and the measured coefficients are $D_{L} / D_{m}=D_{T} / D_{m}=1.3$ after $t=0.4 \mathrm{~s}$ (Fig. 9). Under a $5 \mathrm{kV} / \mathrm{m}$ field, $P e=15$ and dispersion doubles: $D_{T} / D_{m}=2.8$ and the trend appears decreasing, while $D_{L} / D_{m}=2.5$ and the trend is flat. Simulation in the present work predicts $D_{L} / D_{m}=1.1$ in a closed cylinder after $0.4 \mathrm{~s}$, increasing to 1.4 after 1 s (Fig. 10), whereas (Hlushkou et al. 2007) found a fully developed value of $D_{L} / D_{m}=1.25$ for $P e=15$ in an open bulk packing (Hlushkou et al. 2007). There are probably two factors at work. The closed packing has a higher velocity variance than the open packing (Fig. 7). A second factor is the sphere packing effect associated with the small ratio of cylinder to sphere diameter, 6.55. The packing effect does not necessarily manifest as higher velocity variance, but it has a substantial impact on short-time dispersion [cf. Figures 7 and 11 in (Maier et al. 2003)]. Although the experimental and simulated packings had the same ratio of cylinder and sphere diameters, the experimental packing was eight times longer than the simulated cylinder and the packing process may therefore have introduced greater heterogeneity. Bed rearrangement due to fluidization is not thought to be a factor, nor is particle cohesion. Dispersion under PDF is significantly higher than EOF. For $P e=15$, (Hlushkou et al. 2007) found $D_{L} / D_{m}=4$ under PDF in bulk packings, while the correlation (8) for PDF predicts
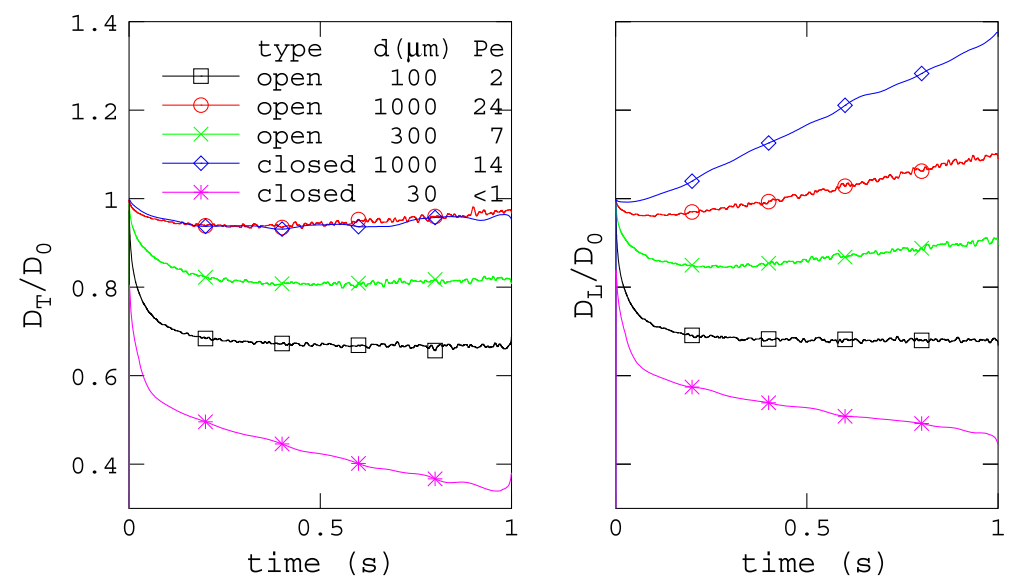

Fig. 10 Transverse and longitudinal dispersion simulated in open and closed sphere packings. Peclet number for closed packings is based on mean backflow velocity 
$D_{L} / D_{m}=8$. Simulations in the present work show that the upward trend in PDF is much stronger than EOF in open and closed packs.

Packed beds of spheres of $d=200 \mu \mathrm{m}$ with $E=2.5 \mathrm{kV} / \mathrm{m}$, yields $P e=2$. The dispersion measurements indicate mild anisotropy with $D_{L} / D_{m}=0.3$ and $D_{T} / D_{m}=0.4$, both slightly larger than their corresponding values in experiments without electroosmotic flow (Fig. 9). For $E=5 \mathrm{kV} / \mathrm{m}, P e=4, D_{T} / D_{m}=0.6, D_{L} / D_{m}=0.7$ and the trends appear flat. In this case, the closed packing is not more dispersive than open packings. For $P e=4$, (Hlushkou et al. 2007) reported $D_{L} / D_{m}=0.8$ for fully developed EOF in an open bulk packing and, in the present work, $D_{L} / D_{m}=0.9$ was obtained after $1 \mathrm{~s}$. Wall effects are thought minor in this case compared to the $1000 \mu \mathrm{m}$ spheres because the larger ratio of cylinder to sphere diameter, 32.75, indicates that the structured region does not propagate into the core of the cylinder. Also, neither fluidization nor particle cohesion is thought to play a role in the packing. In sum, there are no factors that would tend to create major heterogeneities in the closed packing. Dispersion under PDF is higher than EOF, as in the case of $1000 \mu \mathrm{m}$ spheres. For $P e=4$, (Hlushkou et al. 2007) reported $D_{L} / D_{m}=1.25$ for fully developed PDF. A similar value for PDF was obtained in (Maier et al. 2000). The correlation (8) for PDF predicts $D_{L} / D_{m}=3$.

At a sphere diamer of $100 \mu \mathrm{m}$ and $E=2.5 \mathrm{kV} / \mathrm{m}, P e=0.5$. After $0.4 \mathrm{~s}, D_{T} / D_{m}=0.4$ with no trend and $D_{L} / D_{m}=0.6$ with an increasing trend (Fig. 9). For $E=5 \mathrm{kV} / \mathrm{m}$, $P e=1, D_{T} / D_{m}=0.7$, with an increasing trend and $D_{L} / D_{m}=1.6$ with a strongly increasing trend. In this case, the closed packing is more dispersive than both open EOF and PDF. For $P e=1$, (Hlushkou et al. 2007) reported $D_{L} / D_{m}=0.7$ for EOF in an open bulk packing. In the present work, simulation in an open bulk packing yielded $D_{L} / D_{m}=0.7$ after $0.4 \mathrm{~s}$ with a slight decreasing trend. The same value was reported for PDF in (Hlushkou et al. 2007) and (Maier et al. 2000). The correlation (8) for PDF predicts $D_{L} / D_{m}=1.2$. The difference between experiment and simulation is thought to result from heterogeneities in the experimental packing. In $100 \mu \mathrm{m}$ spheres, reverse velocities are probably not large enough to cause local bed movement during the flow experiment. However, cohesive forces are strong enough to balance gravitational forces, and it is conjectured that channels were formed in the experiment by particle aggregation during the packing process.

Further reduction of the sphere size to $d=50 \mu \mathrm{m}$ with $E=2.5 \mathrm{kV} / \mathrm{m}$ further lowers the Peclet number to $P e=0.2$. After $0.4 \mathrm{~s}, D_{T} / D_{m}=0.6$ and the trend is flat to slightly increasing, while $D_{L} / D_{m}=1.0$ and the trend is increasing (Fig. 9). For $E=5 \mathrm{kV} / \mathrm{m}$, $P e=0.4$ and $D_{T} / D_{m}=1.1$ while $D_{L} / D_{m}=2.7$ with a strongly increasing trend. In this case, the evidence for heterogeneities is quite strong. Dispersion in the closed packing is significantly higher than the results for PDF and comparable to the values obtained at much higher $P e$ in $1000 \mu \mathrm{m}$ sphere packs. Based on $P e$, the dispersion coefficient is expected to have subdiffusive behavior, whereas the actual behavior is superdiffusive. The trend is strongly increasing even though the development time is arguably in the asymptotic time frame. The correlation (8) for PDF predicts $D_{L} / D_{m}=1$. In $50 \mu \mathrm{m}$ spheres, reverse flow velocities likely attained in the experiment were locally on the same order as the minimum fluidization. It is conjectured that channels were formed due to particle aggregation during the packing process and that bed movement occurred locally during the flow experiment, further contributing to heterogeneities in the pack structure.

The final sphere size studied was $d=30 \mu \mathrm{m}$. In this size spherical bead pack, for $E=$ $2.5 \mathrm{kV} / \mathrm{m}, P e=0.14$ and dispersion appears isotropic; $D_{T} / D_{m}=D_{L} / D_{m}=0.6$ after 0.4 $\mathrm{s}$ and the trend is weakly increasing (Fig. 9). For $E=5 \mathrm{kV} / \mathrm{m}, P e=0.27$ and $D_{T} / D_{m} \approx$ $D_{L} / D_{m}=0.76$ with an increasing trend. In this case, the evidence for heterogeneities is also strong, but is based more on trends than observed magnitude. Simulation in the present 
work predicts $D_{L} / D_{m}=0.55$ after $0.4 \mathrm{~s}$, and 0.45 after $1 \mathrm{~s}$, with a decreasing trend. The correlation (8) for PDF predicts $D_{L} / D_{m}=0.8$, and while the experimental coefficient is not greater than this value, the trend is increasing. In $30 \mu \mathrm{m}$ spheres, cohesive forces dominate gravitational force, and particle aggregation is likely to have created channels during packing of the experimental cylinder. The backflow velocity likely attained during the experiment is greater than the minimum fluidization velocity, but fluidization of the entire bed is not thought to have occurred because cohesive forces are sufficient to inhibit large-scale particle motion. However, local bed movement may have contributed to channel formation.

\subsubsection{Summary of Results}

EOF dispersion in closed sphere packings is over-predicted by the PDF correlation (8) for larger sphere diameters $(200,1000 \mu \mathrm{m})$ but well predicted or under-predicted at smaller diameters $(30,50,100 \mu \mathrm{m})$. At the smallest diameters, $30 \mu \mathrm{m}$ and $50 \mu \mathrm{m}$, where the measurement time is on the same order as the diffusion timescale, measurements still show increasing trends, indicating the dispersion coefficient is not yet fully developed. The most likely explanation is the existence of channels and cavities in the packing that introduce a length scale larger than the sphere diameter. This explanation is consistent with evidence for pressure-driven flows in columns as well as the evidence on fluidization of particles less than about $100 \mu \mathrm{m}$.

The dispersion coefficient in $50 \mu \mathrm{m}$ and $100 \mu \mathrm{m}$ packings exceeded the PDF correlation to a greater extent than for $30 \mu \mathrm{m}$, in spite of the fact that at $30 \mu \mathrm{m}$, the mean backflow exceeded the minimum fluidization velocity. The $50-\mu \mathrm{m}$ and $100-\mu \mathrm{m}$ packings also exhibited stronger upward trends, indicating larger heterogeneities with longer correlation length. This suggests the possibility that greater cohesive forces in the 30- $\mu \mathrm{m}$ spheres inhibited large-scale bed movement and formation of large channels, compared to slightly larger spheres. Lift and cohesion are both less significant at $50 \mu \mathrm{m}$ and $100 \mu \mathrm{m}$, but may combine to more readily shift and freeze sphere positions in response to local pressure gradients.

The conclusion is that for a given $P e$, dispersion under EOF is greater in a closed packing than an open packing, but less than under PDF, provided the bed is fixed. Where EOF-induced backflow is significant, the fluid pressure can cause structural changes in an unconsolidated medium. These changes may alter the flow and increase dispersion. The potential for structural change appears greatest for particle size less than $100 \mu \mathrm{m}$, where cohesive forces begin to balance gravitational forces (Massimilla and Donsi 1976), and where typical EOF velocities are sufficient for fluidization.

The plots of $D_{L}(t)$, and $D_{T}(t)$ to a lesser extent, go through minima in cases where the Peclet number is transitional between the diffusive and mechanical-dispersion regimes. At short times, $D(t)$ has the dynamics of restricted diffusion, while at longer times, the effects of mechanical dispersion are manifested. Location of the minimum depends on the Peclet number. These results confirm observations in Buhai et al. (2008). The mechanics of the short-time competition between diffusion and advection are discussed in (Codd and Altobelli 2003).

\section{Conclusions}

Fully developed electroosmotic flow in an open cylinder is uniform outside the slip layer. In a sphere packing open to external flow, the EOF velocity is not uniform, but virtually all the fluid is in motion, compared to a pressure-driven flow where the mode is $v=0$. Where the 
mean flow rates are equal, the modes of EOF and PDF have similar amplitude but the EOF distribution is narrower, indicating lower velocity variance than a PDF of comparable force. For a given Peclet number, dispersion under PDF is greater than EOF, consistent with the idea that dispersion is proportional to the velocity variance scaled by the mean.

Electroosmotic flow in a closed cylinder forms a recirculation cell and the velocity distribution has a zero mode corresponding to the shear layer between slip flow along the wall and reverse flow through the interior. Reverse flow is induced by electroosmotic pressure, which reaches a maximum in the case of a porous medium completely closed to external flow. In a closed sphere packing, reverse flow is concentrated in larger pathways and, although porescale recirculation patterns are not uncommon, most of the flow recirculates on a longer length scale. The pressure-driven reverse flow is better fit by an exponential distribution, while the electroosmotic forward flow is better fit by a Gaussian. The reverse flow has greater variance than in the forward flow, analogous to the difference between pure PDF and EOF in an open porous medium. Sphere diameter affects EOF flow and dispersion in closed sphere packings in several ways, including the potential for bed movement in response to back pressure.

Dispersion under EOF is greater in a closed than an open sphere packing, but less than under PDF, provided the bed is fixed. Where induced backflow is significant, EOF has the potential to induce structural changes in an unconsolidated medium. In smaller spheres, reverse flow velocities may reach the minimum fluidization velocity and induce bed movement. Cohesive forces are also significant below this diameter, and while they may inhibit fluidization, particle aggregation may also contribute to heterogeneities in the pack structure, leading to superdiffusive behavior at Peclet numbers otherwise associated with subdiffusive dispersion. The potential for structural change appears greatest for particle size less than $100 \mu \mathrm{m}$, where cohesive forces begin to balance gravitational forces, and where typical EOF velocities are sufficient for fluidization.

Flow curvature and shear gradients are much greater in a closed packing under EOF and place greater demands on the numerical methods. Flow simulations in closed sphere packings exhibited slower convergence and greater continuity errors than in open packings, although qualitative features of the velocity distribution are captured with fairly coarse spatial resolution. Numerical investigations revealed the calculation of the electric field at the sphere surfaces as a source of error with poor grid convergence, and hence, improvements are needed in this direction.

The mechanisms for fluid exchange with dead-end pores differ between EOF and PDF. At short times, the power-law exponents corresponding to dispersion under PDF are larger than EOF, suggesting the possibility of a longer timescale for asymptotic dispersion. However, neither the experimental nor simulation results provide direct evidence on long-time dispersion under EOF and PDF. Longer simulations and theoretical analysis are necessary to deduce this behavior.

Acknowledgments SC and EN acknowledge partial support from U.S. Department of the Army Cooperative Agreement W912HZ-10-2-0018. RM acknowledges an allocation of supercomputer time (CTS140017) from the National Science Foundation XSEDE program. JDS and SLC acknowledge equipment funding from the NSF MRI program and the M. J. Murdock charitable trust.

\section{References}

Acar, Y.B., Alshawabkhek, A.N., Gale, R.J.: Fundamentals of extracting species from soils by electrokinetics. Waste Manag. 13(2), 141-151 (1993)

Adamson, A.W., Gast, A.P.: Physical Chemistry of Surfaces, 6th edn. Wiley, New York (1997) 
Adamson, L.G.: Electrokinetic dewatering, consolidation and stabilization of soils. Eng. Geol. 1(4), 291-304 (1966)

Baerns, M.: Effect of interparticle adhesive forces on fluidization of fine particles. Ind. Eng. Chem. Fundam. 5(4), 508 (1966)

Bear, J.: Dynamics of Fluids in Porous Media. Dover Publications, Inc, New York (2013)

Bijeljic, B., Muggeridge, A.H., Blunt, M.J.: Pore-scale modeling of longitudinal dispersion. Water Resour. Res. 40(11) (2004)

Brenner, H.L., Edwards, D.A.: Macrotransport Processes. Butterworth-Heinemann, Boston (1993)

Buhai, B., Li, Y., Kimmich, R.: Electro-osmotic flow and dispersion in microsystem channel networks and porous glasses: Comparison with pressure-induced transport and ionic currents. Magnetic Resonance Microscopy. Wiley-VCH Verlag GmbH \& Co. KGaA, Weinheim (2008)

Callaghan, P.T.: Principles of Nuclear Magnetic Resonance Microscopy. Clarendon Press, Oxford (1991)

Cardenas, H.E., Struble, L.J.: Electrokinetic nanoparticle treatment of hardened cement paste for reduction of permeability. J. Mater. Civil Eng. 18(4), 554-560 (2006)

Casagrande, L.: Electro-osmotic stabilization of soils. J. Boston Soc. Civil Eng. 39(1), 38-51 (1952)

Chen, H.D., Chen, S.Y., Matthaeus, W.H.: Recovery of the Navier-Stokes equations using a lattice-gas Boltzmann method. Phys. Rev. A 45(8), R5339-R5342 (1992)

Chung, S.F., Wen, C.Y.: Longitudinal dispersion of liquid flowing through fixed and fluidized beds. Aiche J. 14(6), 857 (1968)

Codd, S.L., Altobelli, S.A.: A PGSE study of propane gas flow through model porous bead packs. J. Mag. Resonance 163(1), 16-22 (2003)

Edwards, M.F., Richards, J.F.: Gas dispersion in packed beds. Chem. Eng. Sci. 23(2), 109 (1968)

Fukushima, E.: Nuclear magnetic resonance as a tool to study flow. Ann. Rev. Fluid Mech. 31, 95-123 (1999)

Ghassemi, A., Pak, Ali: Pore scale study of permeability and tortuosity for flow through particulate media using lattice Boltzmann method. Int. J. Numer. Anal. Methods Geomech. 35(8), 886-901 (2011)

Ghosal, S.: Electrokinetic flow and dispersion in capillary electrophoresis. Ann. Rev. Fluid Mech. 38, 309-338 (2006)

He, Q.H., Liu, Y.M., Sun, H.H., Li, E.C.: Capillary array electrophoretic NMR of proteins in biological buffer solutions. J. Mag. Resonance 141(2), 355-359 (1999)

Hlushkou, D., Khirevich, S., Apanasovich, V., Seidel-Morgenstern, A., Tallarek, U.: Pore-scale dispersion in electrokinetic flow through a random sphere packing. Anal. Chem. 79(1), 113-121 (2007)

Hlushkou, D., Seidel-Morgenstern, A., Tallarek, U.: Numerical analysis of electroosmotic flow in dense regular and random arrays of impermeable, nonconducting spheres. Langmuir 21(13), 6097-6112 (2005)

Holz, M., Heil, S.R., Schwab, I.A.: Electrophoretic NMR studies of electrical transport in fluid-filled porous systems. Magn. Reson. Imaging 19(3-4), 457-463 (2001)

Holz, M., Seiferling, D., Mao, X.A.: Design of a new electrophoretic nmr probe and its application to ${ }^{7} \mathrm{li}^{+}$ and ${ }^{1} 33 \mathrm{cs}^{+}$mobility studies. J. Magn. Resonance Ser. 105(1), 90-94 (1993)

Huizenga, D.G., Smith, D.M.: Knudsen diffusion in random assemblages of uniform spheres. Aiche J. 32(1), $1-6(1986)$

Kandhai, D., Hlushkou, D., Hoekstra, A.G., Sloot, P.M.A., Van As, H., Tallarek, U.: Influence of stagnant zones on transient and asymptotic dispersion in macroscopically homogeneous porous media. Phys. Rev. Lett. 88(23), (2002)

Khirevich, S.: High-Performance Computing of Flow, Diffusion, and Hydrodynamic Dispersion in Random Sphere Packings. Philipps-Universitat, Marburg (2010)

Kloeden, P.E.: Numerical Solution of Stochastic Differential Equations, vol. 23. Springer, Berlin (1992)

Lagerblad, B., Vogt, C.: Ultrafine particles to save cement and improve concrete properties CBI report (pp. 40). Stokholm: Cement och Betong Institutet (Swedish Cement and Concrete Research Institute) (2004)

Locke, B.R., Acton, M., Gibbs, S.J.: Electro-osmotic flow in porous media using magnetic resonance imaging. Langmuir 17(22), 6771-6781 (2001)

Mahmoud, A., Olivier, J., Vaxelaire, J., Hoadley, A.F.A.: Electrical field: a historical review of its application and contributions in wastewater sludge dewatering. Water Res. 44(8), 2381-2407 (2010)

Maier, R.S., Bernard, R.S.: Lattice-Boltzmann accuracy in pore-scale flow simulation. J. Comput. Phys. 229(2), $233-255(2010)$

Maier, R.S., Bernard, R.S., Grunau, D.W.: Boundary conditions for the lattice Boltzmann method. Phys. Fluids 8(7), 1788-1801 (1996)

Maier, R.S., Kroll, D.M., Bernard, R.S., Howington, S.E., Peters, J.F., Davis, H.T.: Pore-scale simulation of dispersion. Phys. Fluids 12(8), 2065-2079 (2000)

Maier, R.S., Kroll, D.M., Bernard, R.S., Howington, S.E., Peters, J.F., Davis, H.T.: Hydrodynamic dispersion in confined packed beds. Phys. Fluids 15(12), 3795-3815 (2003) 
Manz, B., Stilbs, P., Jonsson, B., Soderman, O., Callaghan, P.T.: NMR imaging of the time evolution of electroosmotic flow in a capillary. J. Phys. Chem. 99(29), 11297-11301 (1995)

Marcos, Y.K., Ooi, K.T., Yang, C., Wong, T.N.: Frequency-dependent velocity and vorticity fields of electroosmotic flow in a closed-end cylindrical microchannel. J. Micromech. Microeng. 15(2), 301-312 (2005)

Mason, G.: A model of the pore space in a random packing of equal spheres. J. Colloid Interface Sci. 35(2), 279-287 (1971)

Massimilla, L., Donsi, G.: Cohesive forces between particles of fluid-bed catalysts. Powder Technol. 15(2), 253-260 (1976)

O'Brien, R.W.: Electroosmosis in porous materials. J. Colloid Interface Sci. 110(2), 477-487 (1986)

Ottosen, L.M., Christensen, I.V., Rorig-Dalgard, I., Jensen, P.E., Hansen, H.K.: Utilization of electromigration in civil and environmental engineering - Processes, transport rates and matrix changes. J. Environ. Sci. Health A-Toxic/Hazard. Subst. Environ. Eng. 43(8), 795-809 (2008)

Probstein, R.F.: Physicochemical Hydrodynamics: An Introduction. Butterworth-Heinemann, A Division of Reed Publishing, Waltham (1989)

Qian, Y.H., d'Humières, Dominique, Lallemand, Pierre: Lattice BGK models for Navier-Stokes equation. Europhys. Lett. 17(6), 479 (1992)

Rice, C.L., Whitehead, R.: Electrokinetic flow in a narrow cylindrical capillary. J. Phys. Chem. 69(11), 40174024 (1965)

Richardson, J.F., Harker, J.H.: Chemical Engineering: Particle Technology and Separation Processes, vol. 2. Butterworth-Heinemann, Bath (2002)

Schure, M.R., Maier, R.S.: How does column packing microstructure affect column efficiency in liquid chromatography? J. Chromatogr. A 1126(1-2), 58-69 (2006)

Seto, R., Botet, R., Auernhammer, G.K., Briesen, H.: Restructuring of colloidal aggregates in shear flow coupling interparticle contact models with Stokesian dynamics. Eur. Phys. J. E 35(12), (2012)

Shiota, I., M, Y.: Functionally Graded Materials 1996. Elsevier, Amsterdam (1997)

Sterling, J.D., Chen, S.Y.: Stability analysis of lattice Boltzmann methods. J. Comput. Phys. 123(1), 196-206 (1996)

Stilbs, P., Furo, I.: Electrophoretic NMR. Curr. Opin. Colloid Interface Sci. 11(1), 3-6 (2006)

Sullivan, S.P., Sani, F.M., Johns, M.L., Gladden, L.F.: Simulation of packed bed reactors using lattice Boltzmann methods. Chem. Eng. Sci. 60(12), 3405-3418 (2005)

Tallarek, U., Rapp, E., Scheenen, T., Bayer, E., Van As, H.: Electroosmotic and pressure-driven flow in open and packed capillaries: velocity distributions and fluid dispersion. Anal. Chem. 72(10), 2292-2301 (2000). doi:10.1021/ac991303i

Wolf-Gladrow, D.A.: Lattice-gas cellular automata and Boltzmann models: an introduction. Springer, Berlin (2000)

Xuan, X.C., Xu, B., Sinton, D., Li, D.Q.: Electroosmotic flow with Joule heating effects. Lab Chip 4(3), 230-236 (2004). doi:10.1039/b315036d

Yao, S.H., Santiago, J.G.: Porous glass electroosmotic pumps: theory. J. Colloid Interface Sci. 268(1), 133-142 (2003) 